\title{
On the existence of Weibel instability in a magnetized plasma. I. Parallel wave propagation
}

\author{
M. Lazar, ${ }^{1,2, a)}$ R. Schlickeiser, ${ }^{2}$ and S. Poedts ${ }^{1}$ \\ ${ }^{1}$ Center for Plasma Astrophysics, Celestijnenlaan 200B, 3001 Leuven, Belgium \\ ${ }^{2}$ Institut für Theoretische Physik, Lehrstuhl IV: Weltraum- und Astrophysik, Ruhr-Universität Bochum, \\ D-44780 Bochum, Germany
}

(Received 22 September 2008; accepted 28 December 2008; published online 30 January 2009)

\begin{abstract}
In a uniform static magnetic field $\left(B_{0}\right)$ the nonresonant mechanism of Weibel instability is certainly affected, because the free energy stored in the temperature anisotropy (e.g., $T_{\perp}>T_{\|}$, where $\perp$ and $\|$ denote directions relative to the background magnetic field) can drive the parallel whistler instability due to the cyclotron resonance with plasma particles. However, it has already been shown that the nonresonant mechanism should exist and drive the whistler instability, at least, for anisotropic distribution functions with no parallel thermal velocity spread. Here the investigation is extended to a magnetized plasma with a bi-Maxwellian temperature anisotropy. New frequency limits are introduced and the necessary physical conditions are provided: The nonresonant whistler instabilities have in general small frequencies, smaller than the growth rate, $\omega_{r}<\omega_{i}$, and for a large temperature anisotropy, the nonresonant instabilities are limited to a $\beta_{\perp}=8 \pi n_{0} T_{\perp} / B_{0}^{2}>1$ regime. These criteria restricts in general the magnetic field to strengths less than a threshold value proportional to the square root of the temperature anisotropy. In the presence of an ambient magnetic field, the growth rate of the nonresonant instability is reduced and the large wavelengths are stabilized. () 2009 American Institute of Physics. [DOI: 10.1063/1.3072976]
\end{abstract}

\section{INTRODUCTION}

Space plasmas are magnetized and many processes such as magnetic compression or expansion lead to an anisotropic temperature distribution of the electrons (see Ref. 1, and references therein). Heating plasma electrons perpendicular to the background magnetic field $\left(\mathbf{B}_{0}\right)$ does not change their mobility considerably, whereas parallel heating enables the particles to move more rapidly along $\mathbf{B}_{0}$. Thus, parallelheated electrons may leave the region of energization more quickly, implying that the instabilities driven by $T_{\perp}>T_{\|}$ should be more often observed in space plasmas. Such anisotropic structures of plasma are unstable and release the available free energy by exciting specific plasma waves and instabilities.

In a magnetized plasma, the electromagnetic branch of parallel modes includes the whistler modes with frequencies below the electron gyrofrequency, $\Omega_{i}<\omega \ll\left|\Omega_{e}\right|=\Omega$, where $\Omega_{e, i}$ are the electron and ion gyrofrequencies, respectively, and the electron cyclotron modes with higher frequencies, in the vicinity of the electron gyrofrequency, $\omega \lesssim \Omega$. These modes have subluminal phase velocities, $\omega / k<c$ ( $c$ is the speed of light in vacuum), but frequencies sufficiently high to neglect the ions reaction. In the first attempts of describing nonrelativistically the high frequency electromagnetic instabilities generated along the stationary ambient magnetic field by a plasma temperature anisotropy, it has been found ${ }^{2,3}$ that, for a gyrotropic plasma with $T_{\perp}>T_{\|}$, the instability is restricted to small wave numbers, less than a maximum cutoff value, $k \leqslant k_{M}$, given by

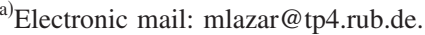

$$
k_{M} \simeq\left[A \omega_{p}^{2}+A^{2} \Omega^{2} /(A+1)^{2}\right]^{1 / 2} / c,
$$

where $A=T_{\perp} / T_{\|}-1$. Sudan ${ }^{2}$ also launched the idea that this instability could exist even for the opposite case of $T_{\perp}<T_{\|}$, when the magnetic field is sufficiently strong satisfying the condition $\Omega^{2} / \omega_{p}^{2}>1 / A(A+1)$. This conclusion was, however, suspected to be erroneously obtained because in this case, the phase velocity of the unstable mode is superluminal, $v_{\mathrm{ph}}>c$ (where $c$ is the speed of light in vacuum), and the superluminal modes cannot interact resonantly with plasma particles being almost stable. As a matter of fact, 2 years latter $\operatorname{Sudan}^{4}$ provided a relativistically correct approach, limiting the plasma particle velocities to values less than the speed of light in vacuum, and showing rigorously that, even for low nonrelativistic plasma temperatures, the high frequency electromagnetic instability along the ambient magnetic field exists only for $T_{\perp}>T_{\|}$.

The relativistic effects tend to reduce the (temporal) growth rate of the whistler instability, but they are not sufficient by themselves to stabilize this mode. ${ }^{5}$ Thus, the stability properties of the whistler Weibel-type modes have been examined in detail for relativistically hot electron-positron plasmas, ${ }^{6}$ which are supposed to be present in many astrophysical sources of synchrotron radiation. Considering two simple cases of water-bag or smooth anisotropic distribution functions it was shown that the presence of an ambient magnetic field reduces the growth rate and can even suppress the instability, when the magnetic field is stronger than a threshold value.

There is, however, a sustained interest for understanding the properties of the whistler instability in the nonsymmetric electron-ion plasmas at low nonrelativistic temperatures. ${ }^{7-9}$ Analytical approximative forms of the linear growth rates 
have been derived for different anisotropic distribution functions. ${ }^{2-4,10,11}$ Kennel and Petschek ${ }^{10}$ have used the pitch angle scattering by this mode as a source of precipitating electrons in space plasma magnetosphere. The first accurate dispersion curves have been obtained numerically, ${ }^{5,12,13}$ and have been used to evaluate the relaxation time of different temperature anisotropies for various static magnetic fields. ${ }^{14}$ The numerical experiments have proved that both linear saturation and isotropization $\left(T_{\perp} \simeq T_{\|}\right)$occur when the growth rate is of the order of the particle trapping frequency. ${ }^{15,16}$

The electron whistler instability has been regarded as the natural extension of the Weibel instability ${ }^{17}$ to the case of a magnetized plasma. ${ }^{18}$ However, the physical mechanisms through which the free energy of plasma particles is extracted and transferred to these instabilities can be completely different: the Weibel instability is a magnetic instability essentially nonresonant with plasma particles and induced by the Biot-Savart current-current interaction between the microscopic particle streams arising in a plasma with a temperature anisotropy, ${ }^{19,20}$ while the instability of the whistler mode is fed by the resonant plasma particles, when their Doppler-shifted frequency is closed to the cyclotron frequency of the electrons. The presence of the background magnetic field will affect the generation mechanism of the Weibel instability, that should, however, remain viable, at least for a weak magnetic field, or in a plasma with a sufficiently strong temperature anisotropy. The numerical simulations have confirmed that an ambient magnetic field $\mathbf{B}_{0}$ tends to suppress the instability with the growing magnetic field perpendicular to $\mathbf{B}_{0}{ }^{21}$

For the whistler instability, the existence of both regimes, resonant and nonresonant (or reactive), has already been perceived, ${ }^{3-5,8}$ and the first rigorous analysis has been provided by Lee. ${ }^{3}$ He tried to make a clear distinction between the resonant and the nonresonant regimes of the whistler instability by using idealistic forms of the anisotropic distribution functions with no parallel thermal velocity spread, which, by simple intuition, must drive the nonresonant instability of the Weibel-type. However, in these cases, for $\omega_{r} \sim \Omega$, the cyclotron resonance (no Doppler shift) can still resonantly drive the electromagnetic instability as long the electrons are magnetically confined. In the present paper we propose an additional criterion for the existence of the nonresonant instabilities of the Weibel-type in the presence of a stationary magnetic field. For large temperature anisotropies and negligible thermal spread along the background magnetic field, the magnetic fields generated by the Weibel mechanism can exist and grow only for a thermal pressure larger than the magnetic pressure, that means, for a plasma with a large perpendicular beta (nonconfined)

$$
\beta_{\perp}=\frac{8 \pi n_{0} T_{\perp}}{B_{0}^{2}}=\left(\frac{\omega_{p}}{\Omega}\right)^{2}\left(\frac{v_{\mathrm{th}, \perp}}{c}\right)^{2}>1
$$

If this condition is satisfied, then the electrons are able to bunch under the action of a magnetic perturbation, and induce the growing of the nonresonant instability.

Here we extend the investigation to a less restrictive

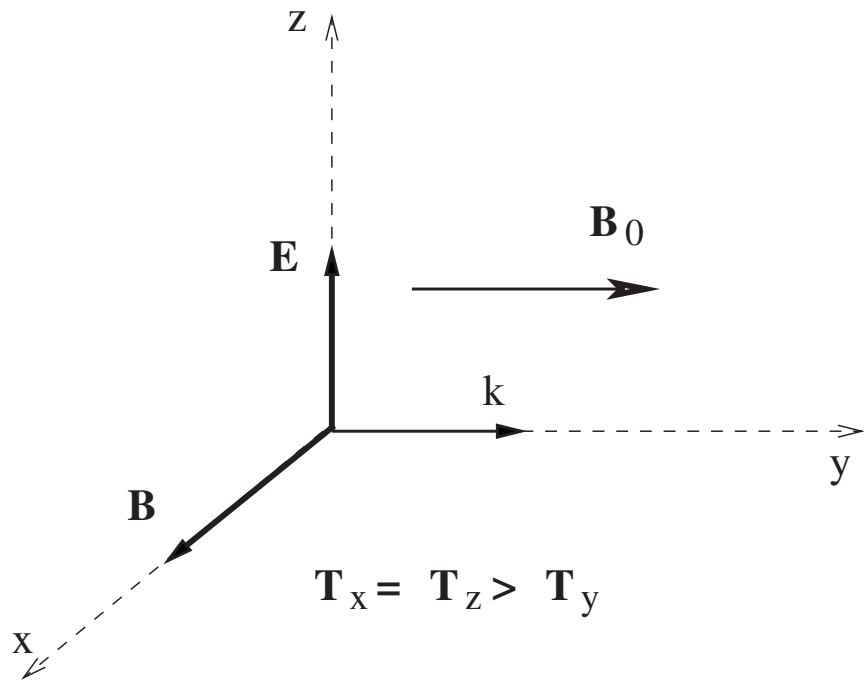

FIG. 1. The orientation of the ambient magnetic field $\mathbf{B}_{0}$ parallel to the $y$-axis, and the temperature anisotropy, $T_{x}=T_{z}>T_{y}$ so that the instability propagates along the magnetic field, $\mathbf{k} \| \mathbf{B}_{0}$.

plasma with a bi-Maxwellian distribution, where the resonant plasma particles present an additional source for the whistler instability. The existence of this instability is conditioned by $A=T_{\perp} / T_{\|}-1>0$, and the unstable wave numbers are limited by Eq. (1). ${ }^{4}$ In order to identify the Weibel-type instability, we have to make a distinction between the resonant and nonresonant regimes of the whistler instability, and therefore, we proceed to a parametric analysis, function of the wave frequency, as well as the level of anisotropy and the strength of the magnetic field.

The dispersion relation of parallel electromagnetic modes is provided in Sec. II in terms of the plasma dispersion function. ${ }^{22}$ In Sec. III we look for the unstable modes which are far from being resonant with the electrons, considering, as suggested in previous works, ${ }^{5,8}$ those modes for which the argument of the plasma dispersion function is either very large $(f \gg 1)$ or very small $(f \ll 1)$. The exact numerical solutions are compared in order to confirm the criterion introduced in Eq. (2) for the existence of the nonresonant instabilities of the Weibel-type. The limit case of a very large anisotropy is associated with the Weibel-type filamentation instability, and it is discussed in Sec. IV. We summarize the results and give our conclusions in the last section.

\section{PARALLEL TRANSVERSE MODES, $\mathbf{k}_{\|} \mathbf{B}_{0}$}

We assume that only the electrons exhibit a thermal anisotropy of a bi-Maxwellian-type,

$$
F\left(v_{x}, v_{y}, v_{z}\right)=\frac{1}{\pi^{3 / 2} v_{\mathrm{th}}^{2} v_{\mathrm{th}, y}} \exp \left[-\frac{v_{x}^{2}+v_{z}^{2}}{v_{\mathrm{th}}^{2}}-\frac{v_{y}^{2}}{v_{\mathrm{th}, y}^{2}}\right],
$$

and therefore consider only their contribution to the wave dispersion. In Fig. 1 the orientation of the electromagnetic unstable mode is given with respect to the Cartesian coordinate system: the temperature anisotropy is biaxial, $T_{x}=T_{z}$ $>T_{y}$, and drives the instability along the ambient magnetic field $\mathbf{B}_{0}$, which is parallel to the $y$-axis. The dispersion rela- 
tion of the parallel transverse modes has been derived by Weibel, ${ }^{17}$

$$
1-\frac{k^{2} c^{2}}{\omega^{2}}+\frac{\omega_{p}^{2}}{\omega^{2}}\left[A+\left(\frac{\omega}{k v_{\mathrm{th}, \|}}+A f\right) Z(f)\right]=0
$$

in terms of the plasma dispersion function $Z(f),{ }^{22}$ with the argument

$$
f=\frac{\omega-\Omega}{k v_{\text {th, }}}
$$

and the temperature anisotropy

$$
A=\frac{v_{\mathrm{th}, \perp}^{2}}{v_{\mathrm{th}, \|}^{2}}-1=\frac{T_{\perp}}{T_{\|}}-1>0 .
$$

The plasma frequency $\omega_{p}=\left(4 \pi n e^{2} / m\right)^{1 / 2}$, and the absolute value of the electron gyrofrequency $\Omega=|e| B_{0} /(m c)$ are introduced above.

Weibel has chosen to describe the instability neglecting the influence of the background magnetic field $(\Omega \simeq 0)$, and in the limit of a large argument, $f \gg 1$, of $Z$. Here, we consider plasma magnetized, $\Omega \neq 0$, and look for the unstable solutions in the both limit cases: ${ }^{23}$ for small arguments, $f$ $\ll 1$, an important number of resonant plasma particles still exist making the electromagnetic mode unstable, while for large arguments, $f \gg 1$, if the instability exists, it is expected to be nonresonant, ${ }^{5,8}$ and therefore identified as a Weibeltype instability. These conditions are not sufficient, and we need new criteria to fix the limits of the nonresonant instability.

\section{NONRESONANT WHISTLER (WEIBEL-TYPE) INSTABILITY}

The resonant unstable modes are usually described by those arguments of the plasma dispersion function in Eq. (5) which are comparable to unity or less, ${ }^{8}$

$$
f=\frac{\omega-\Omega}{k v_{\text {th, }, \|}} \lesssim 1 .
$$

It is therefore suggested that the nonresonant instabilities should be identified in the opposite limits of the very large ${ }^{8}$ and, as we suppose here, the very small arguments of plasma dispersion function. In these limits, the number of resonant plasma particles is expected to be small or even negligible. These limits are, however, not sufficient for making a clear distinction between the resonant and nonresonant instabilities because the correct cyclotron resonance relation $f_{r}=\left(\omega_{r}\right.$ $-\Omega) /\left(k v_{\text {th, }}\right) \sim 1$ involves only the real part of the frequency. Therefore, solving numerically the dispersion relation (3) we also expect to find nonresonant unstable modes satisfying condition (7).

In addition, the frequency of the nonresonant instabilities is expected to be finite but small, smaller than the growth rate, $\omega_{r}<\omega_{i}$, because the Weibel instability in the absence of a stationary magnetic field is purely growing, $\omega_{r}=0$, without any oscillation in time. One such criterion seems to be confirmed in Figs. 2-7, where the growth rates of the parallel

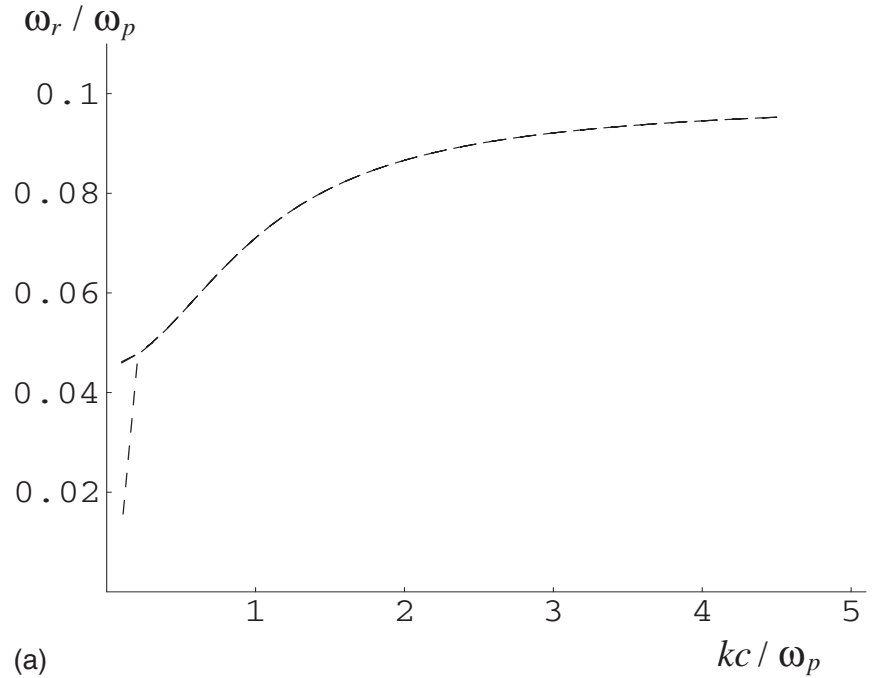

$\omega_{i} / \omega_{p}$

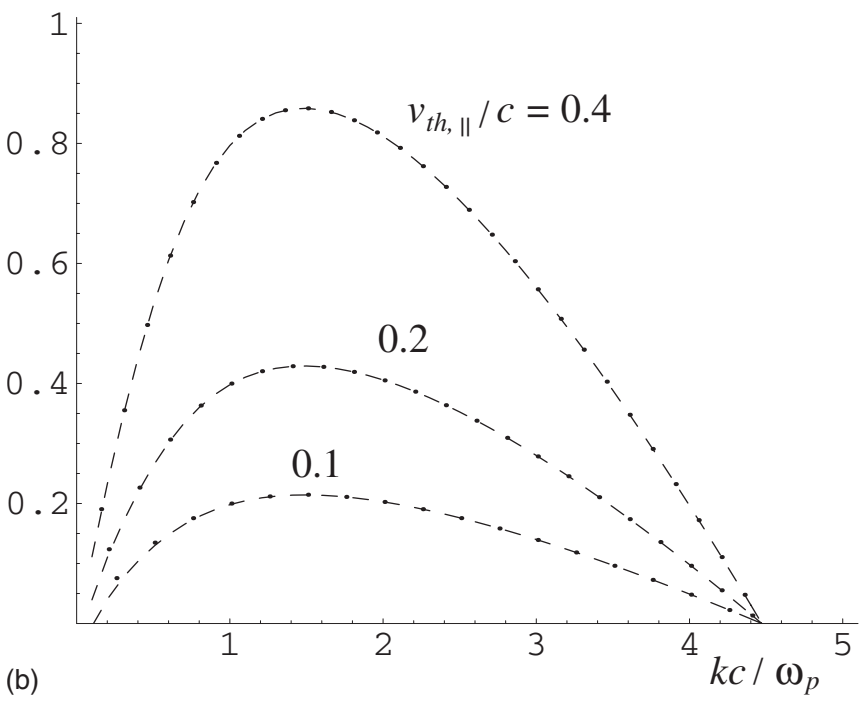

FIG. 2. Comparison of the nonresonant Weibel-type solutions (dotted lines) in the absence of the stationary magnetic field $(\Omega=0)$ and the exact solutions of Eq. (3) (dashed lines) for a weak magnetic field, $\Omega / \omega_{p}=0.1$, a large temperature anisotropy $A=20$ and three large values of parallel thermal velocity $v_{\text {th, }, l} / c=0.1,0.2,0.4$. The wave frequency (a) is smaller and much smaller than the growth rate (b), $\omega_{r} \ll \omega_{i}$. These are nonresonant instabilities in the range of the whistler and cyclotron frequencies.

electromagnetic modes approach very well those of the Weibel instability $\left(B_{0}=0\right)$, as long the wave frequency is smaller than the growth rate.

\section{A. Large arguments: $f \gg 1$}

First, let us consider large arguments of the plasma dispersion function,

$$
f=\frac{|\omega-\Omega|}{k v_{\text {th, }, \|}} \gg 1,
$$

which corresponds to small temperatures along the background magnetic field direction, and, in general, to large temperature anisotropies, $A=\left(v_{\mathrm{th}, \perp} / v_{\mathrm{th}, \|}\right)^{2}-1 \gg 1$. We therefore expect to obtain perfect agreement with the dispersion relations derived in Ref. 3 for distribution functions with no 


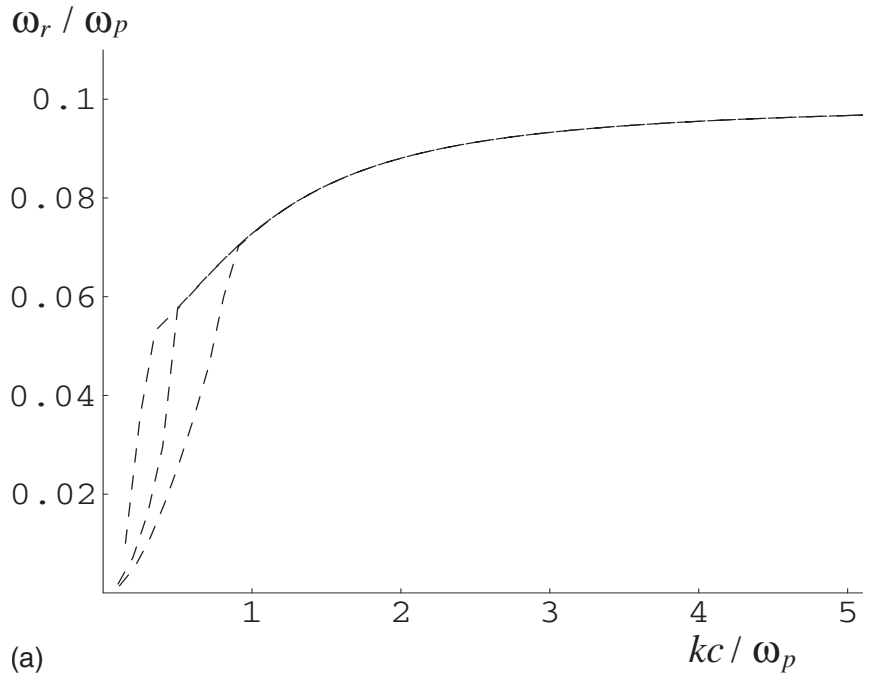

(a)

$\omega_{i} / \omega_{p}$

0.16

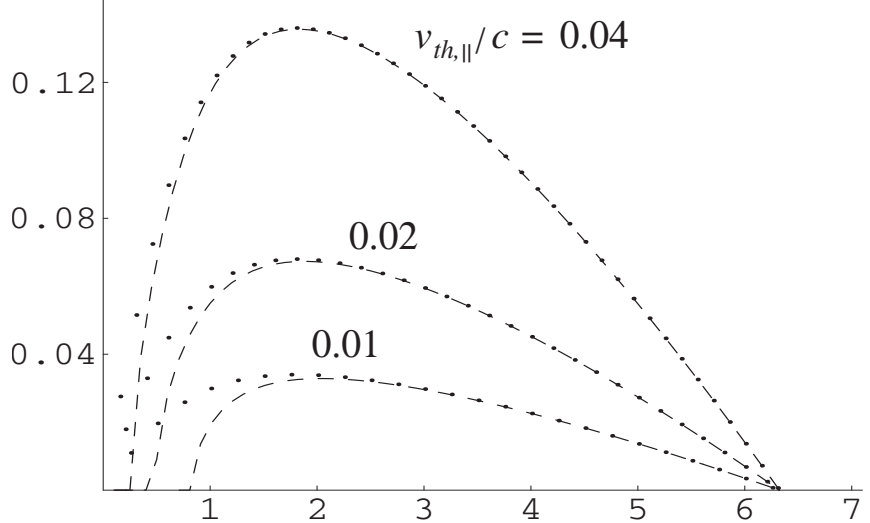

(b)

$k c / \omega_{p}$

FIG. 3. Comparison of the nonresonant Weibel-type solutions (dotted lines) in the absence of the stationary magnetic field $(\Omega=0)$ and the exact solutions of Eq. (3) (dashed lines) for a moderately weak magnetic field, $\Omega / \omega_{p}=0.1$, a very large temperature anisotropy $A=40$ and three very small values of parallel thermal velocity $v_{\mathrm{th}, \|} / c=0.01,0.02,0.04$. The wave frequency (a) is smaller or comparable with the growth rate (b), $\omega_{r} \lesssim \omega_{i}$. The nonresonant modes have the growth rate larger or comparable with the wave frequency and arise when the perpendicular thermal velocity is large enough satisfying $v_{\mathrm{th}, \perp}=\sqrt{A+1} v_{\mathrm{th}, \|}>\left(\Omega / \omega_{p}\right) c$.

parallel thermal velocity spread, but the nonresonant Weibel mechanism will drive the electromagnetic instabilities only for plasmas with a large perpendicular beta, $\beta_{\perp}>1$.

In this case, the asymptotic second order expansion of $Z(f \gg 1)$ in Eq. (4) yields

$$
\frac{\omega^{2}-k^{2} c^{2}}{\omega_{p}^{2}}-\frac{\omega}{\omega-\Omega}-\frac{1}{2}\left(A+\frac{\omega}{\omega-\Omega}\right) \frac{k^{2} v_{\mathrm{th}, \|}^{2}}{(\omega-\Omega)^{2}}=0 .
$$

Now we can neglect $\omega^{2} \ll k^{2} c^{2}$ because the whistler modes are subluminal, with a phase velocity $v_{\text {phase }}=\omega / k<c$, where $c$ is the speed of light in vacuum. The superluminal waves with $v_{\text {phase }}=\omega / k>c$ do not resonate with plasma particles and are, in general, stable. ${ }^{24}$ Perhaps the nonresonant mechanism of Weibel could make the superluminal waves unstable but this analysis must be addressed to a relativistic approach.
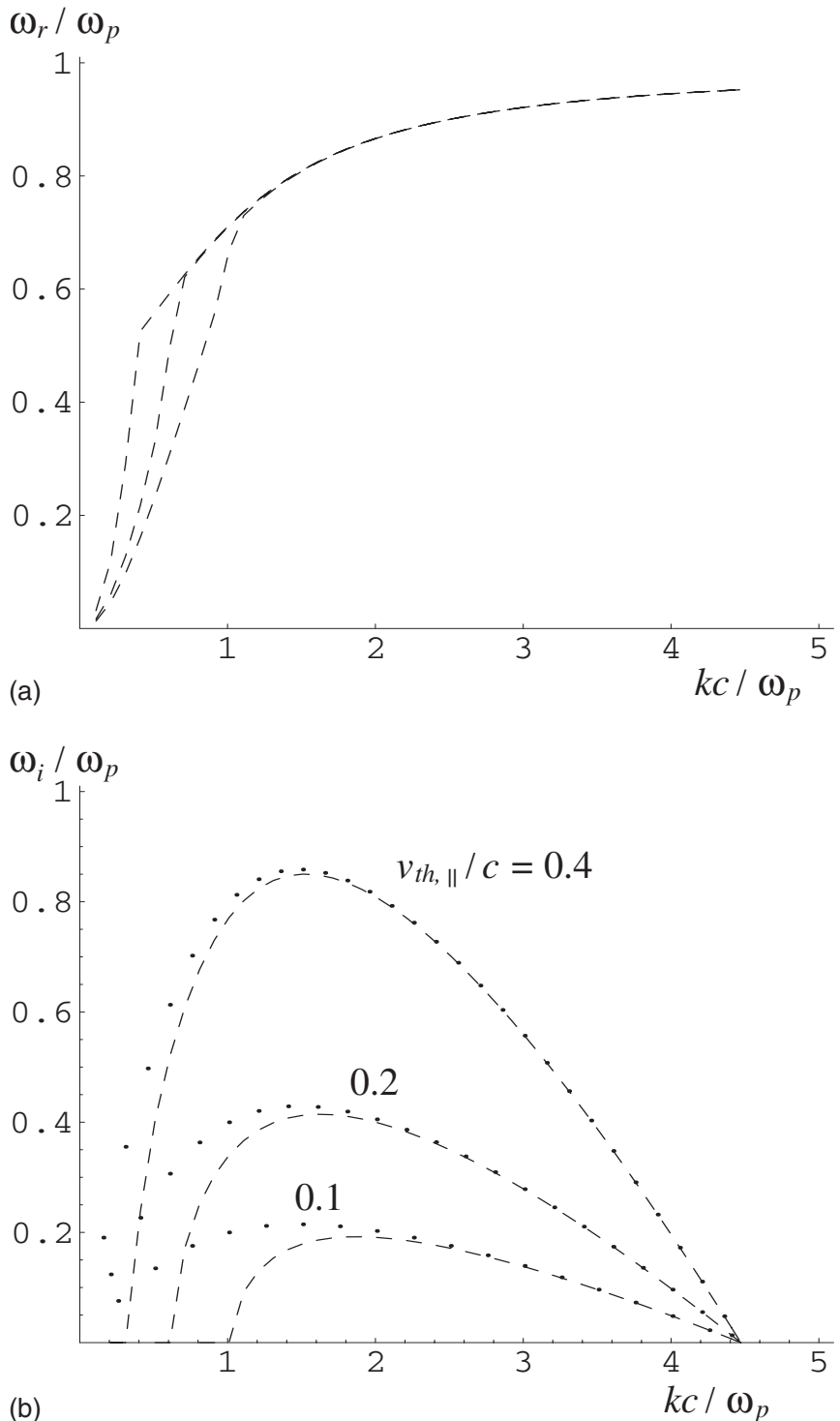

FIG. 4. Comparison of the nonresonant Weibel-type solutions (dotted lines) in the absence of the stationary magnetic field $(\Omega=0)$ and the exact solutions of Eq. (3) (dashed lines) for a strong magnetic field, $\Omega / \omega_{p}=1$, a large temperature anisotropy $A=20$, and three values of parallel thermal velocity $v_{\text {th }, \|} / c=0.1,0.2,0.4$. The wave frequency (a) is comparable to the growth rate (b), $\omega_{r} \sim \omega_{i}$. These instabilities are resonant for low whistler frequencies, and nonresonant for cyclotron frequencies.

Assuming a sufficiently large anisotropy, so that $A \gg \omega / \mid \omega$ $-\Omega$, we find that Eq. (9) admits growing solutions with a finite real frequency

$$
\omega_{r}=\Omega\left[1-\frac{\omega_{p}^{2}}{2\left(\omega_{p}^{2}+k^{2} c^{2}\right)}\right]
$$

which agrees exactly with that obtained by $\mathrm{Lee}^{3}$ [see Eq. (16)], and vanishes in the absence of the magnetic field $(\Omega$ $=0 \rightarrow \omega_{r}=0$ ).

For wave numbers larger than a minimum cutoff value $k \geqslant k_{m}$, given by

$$
k_{m}^{2}=\frac{\omega_{p}^{2}}{2 c^{2}}\left[\left(1+\frac{2}{A} \frac{\Omega^{2}}{\omega_{p}^{2}} \frac{c^{2}}{v_{\mathrm{th}, \|}^{2}}\right)^{1 / 2}-1\right],
$$

we find the growth rate 

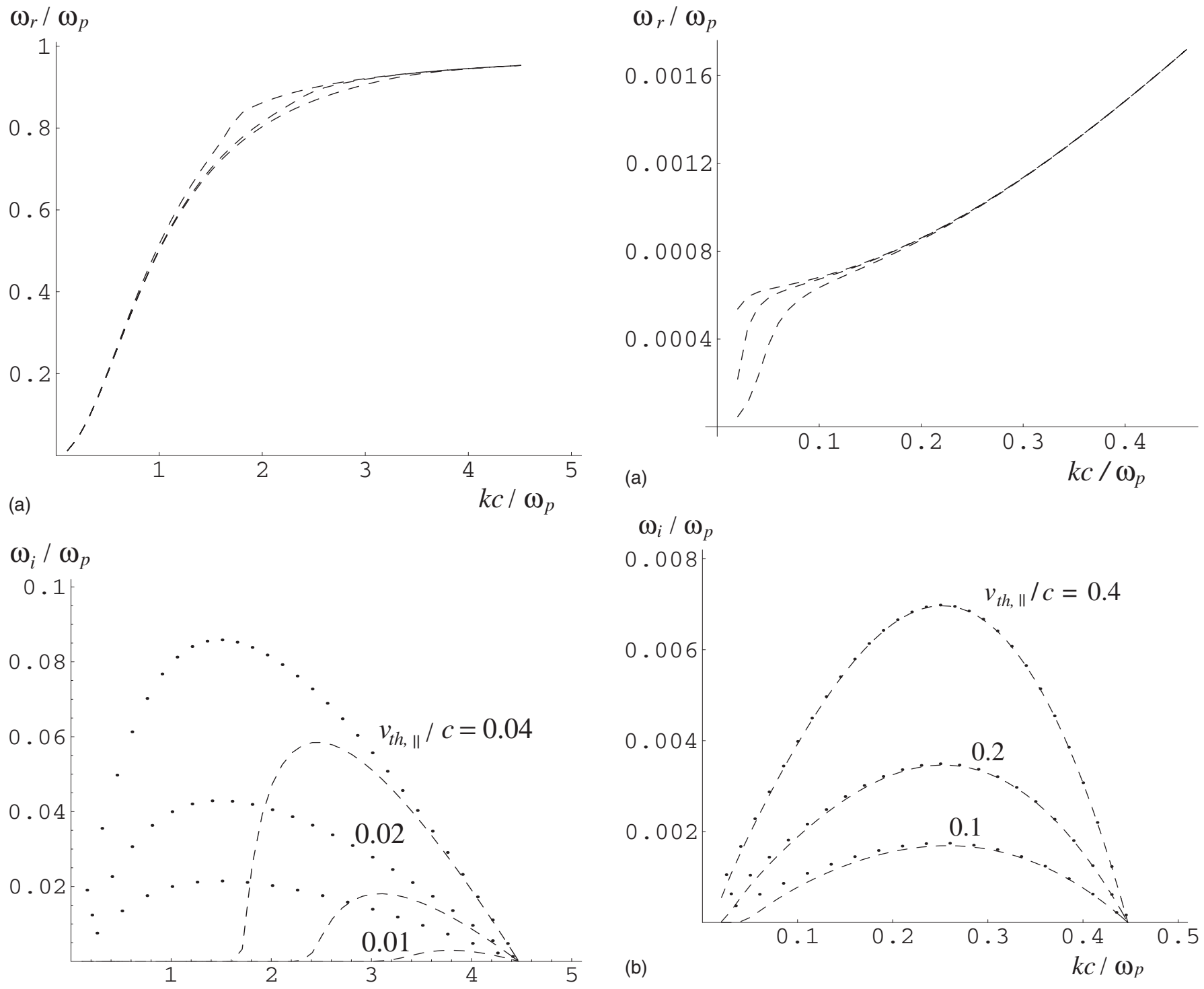

(b)

FIG. 5. Comparison of the nonresonant Weibel-type solutions (dotted lines) in the absence of the stationary magnetic field $(\Omega=0)$ and the exact solutions of Eq. (3) (dashed lines) for a strong magnetic field, $\Omega / \omega_{p}=1$, a large temperature anisotropy $A=20$, and three small values of parallel thermal velocity $v_{\text {th, },} / c=0.01,0.02,0.04$. The wave frequency (a) is much larger than the growth rate (b), $\omega_{r} \gg \omega_{i}$. These instabilities are resonant, and only for larger parallel temperatures, they become nonresonant towards the maximum cutoff wave number.

$$
\omega_{i}=\omega_{p} \frac{\left[2 A k^{2} v_{\mathrm{th}, \|}^{2}\left(\omega_{p}^{2}+k^{2} c^{2}\right)-\Omega^{2} \omega_{p}^{2}\right]^{1 / 2}}{2\left(\omega_{p}^{2}+k^{2} c^{2}\right)} .
$$

In the limit of a very large anisotropy, $A \gg 1$, it reduces exactly to that obtained by Lee, ${ }^{3}$

$$
\omega_{i}=\omega_{p} \frac{\left[2 k^{2} v_{\mathrm{th}, \perp}^{2}\left(\omega_{p}^{2}+k^{2} c^{2}\right)-\Omega^{2} \omega_{p}^{2}\right]^{1 / 2}}{2\left(\omega_{p}^{2}+k^{2} c^{2}\right)} .
$$

Here in Eq. (11) we provide the correct form of the minimum cutoff wave number derived for the first time by Lee, ${ }^{3}$ but with a typographical error [see Eq. (14) in Lee ${ }^{3}$ ]. In the absence of an ambient magnetic field the growth rate in Eq. (12) simplifies exactly to that derived by Weibel, ${ }^{17}$ viz.,

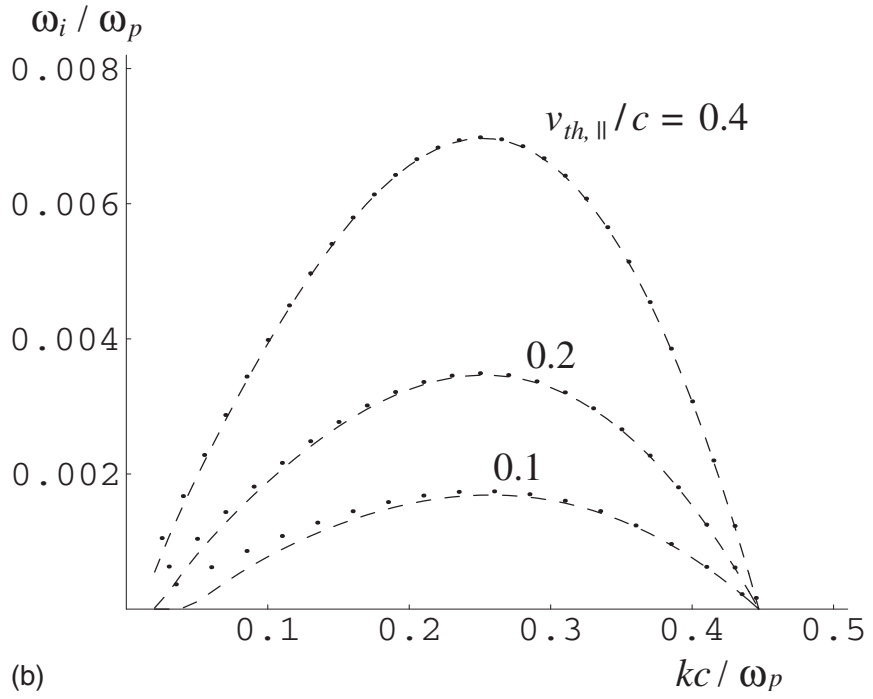

FIG. 6. Comparison of the nonresonant Weibel-type solutions (dotted lines) in the absence of the stationary magnetic field $(\Omega=0)$ and the exact solutions of Eq. (3) (dashed lines) for a very weak magnetic field, $\Omega / \omega_{p}=0.01$, a small temperature anisotropy $A=0.2$, and three values of parallel thermal velocity $v_{\text {th,\| }} / c=0.1,0.2,0.4$. The wave frequency (a) is much smaller than the growth rate $(\mathrm{b}), \omega_{r} \ll \omega_{i}$. These whistler instabilities are Weibel-type (nonresonant).

$$
\omega_{i}=k\left(\frac{A T_{\|}}{m}\right)^{1 / 2} \frac{\omega_{p}}{\left(\omega_{p}^{2}+k^{2} c^{2}\right)^{1 / 2}} \simeq k\left(\frac{T_{\perp}}{m}\right)^{1 / 2} \frac{\omega_{p}}{\left(\omega_{p}^{2}+k^{2} c^{2}\right)^{1 / 2}} .
$$

In the presence of a stationary magnetic field the growth rate is reduced, but for large wave numbers, $k \rightarrow \infty$, both forms (12) and (14) approach the same maximum

$$
\omega_{i}^{\max }=\frac{\omega_{p} v_{\mathrm{th}, \|}}{c} \sqrt{\frac{A}{2}} .
$$

The range of the unstable wave numbers is also reduced, so that the long wavelength modes with $k<k_{m}$ are stabilized by the background magnetic field.

On the other hand, the dispersion relations from above, e.g., Eqs. (10) and (12) are not relevant for small frequencies 


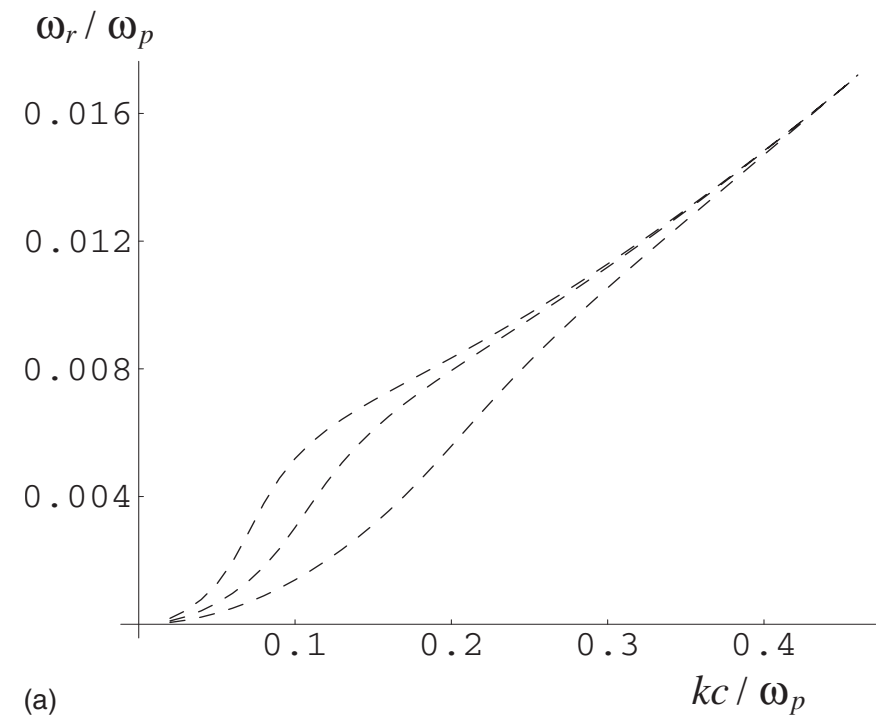

$\omega_{i} / \omega_{p}$

0.012
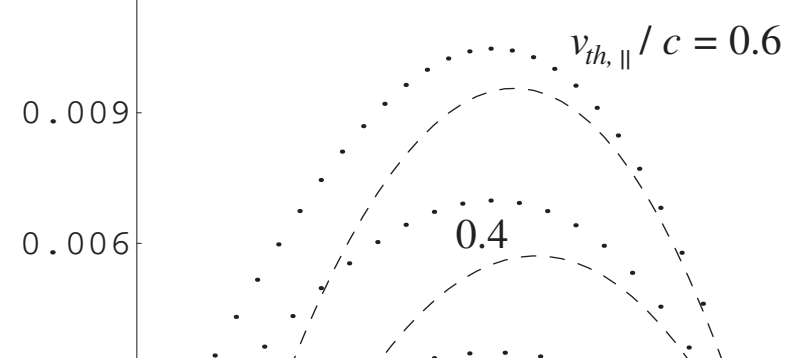

0.003

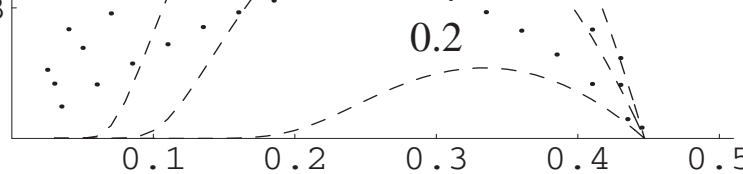

(b)

$k c / \omega_{p}$

FIG. 7. Comparison of the nonresonant Weibel-type solutions (dotted lines) in the absence of the stationary magnetic field $(\Omega=0)$ and the exact solutions of Eq. (3) (dashed lines) for a moderately weak magnetic field, $\Omega / \omega_{p}=0.1$, a small temperature anisotropy $A=0.2$, and three values of parallel thermal velocity $v_{\text {th, } / /} / c=0.2,0.4,0.6$. The wave frequency (a) becomes comparable and even larger than the growth rate (b), $\omega_{r} \gtrsim \omega_{i}$. These whistler instabilities are resonant with the electrons.

and wave numbers, for $k \rightarrow 0$ we get $\omega_{r} \rightarrow \Omega / 2$ and $\omega_{i}<0$. But if we start again from the dispersion relation (9) and restrict to low frequencies in the standard range of whistler waves, $\omega_{r} \ll \Omega$, we find the wave frequency ${ }^{1}$

$$
\omega_{r}=\Omega \frac{k^{2} c^{2}}{\omega_{p}^{2}}\left[1+A \frac{\omega_{p}^{2}}{\Omega^{2}} \frac{v_{\mathrm{th}, \|}^{2}}{c^{2}}\right],
$$

and a very small growth rate, $\omega_{i} \rightarrow 0$. It can be confirmed if we look to the exact numerical solutions plotted here in Figs. 2-5, or in Ref. 1, that Eq. (16) describes the first parabolic branch of the low frequency wave dispersion, where the growth rate is negligibly small.

We have derived the dispersion relations in the limit of large temperature anisotropies but with a finite parallel thermal spread. Our relations agree with those provided by $\mathrm{Lee}^{3}$ in the limit of a negligible parallel thermal spread. The in- equality (8) does not provide us the limits and plasma parameters relevant for the nonresonant instability, but it can be used to derive the analytical forms of the frequency and the growth rate, and to make estimations in some limits.

As we have shown in the Introduction, the nonresonant mechanism of Weibel (intuitively described by Medvedev and Loeb $^{20}$ ) can drive the instability only at $\beta_{\perp}>1$. In such plasmas, the perpendicular kinetic energy is large enough to overcome the magnetic force energy, and the electrons will be able to bunch under the action of a magnetic perturbation, and then induce the growing whistler mode. This new criterion can be confirmed by a comparative evaluation of the exact numerical solutions of Eq. (4), as they are illustrated in Figs. 2-5 for large and very large temperature anisotropies. The wave frequency and the growth rates of the whistler instability are plotted with dashed lines, while the Weibel growth rate is plotted with the dotted line.

The nonresonant character of the whistler modes can be identified in Figs. 2 and 3, where the growth rates approach quite well those of the nonresonant Weibel instability, and this only happens for plasma parameters satisfying condition (2). This condition ensures the existence of the nonresonant instabilities, and, at the same time, a maximum growth rate (15) larger (or even much larger) than the wave frequency, $\omega_{i}^{\max }>\omega_{r}$.

Otherwise, if the threshold imposed in Eq. (2) for the stationary magnetic field is surpassed,

$$
\Omega \equiv \frac{|e| B_{0}}{m c}>\Omega_{t}=\omega_{p} \frac{v_{\mathrm{th}, \perp}}{c}=\omega_{p} \sqrt{A+1} \frac{v_{\mathrm{th}, \|}}{c},
$$

the growth rates of the whistler instability depart considerably from those of the Weibel instability. These instabilities are illustrated in Figs. 4 and 5, where the magnetic field is too strong and make them resonant with the electrons. In this case, condition (2) is not satisfied and furthermore, the wave frequency becomes comparable or larger than the growth rate.

\section{B. Small arguments: $f \ll 1$}

Here we add the case of very small arguments of the plasma dispersion function,

$$
f=\frac{|\omega-\Omega|}{k v_{\text {th }, \|}} \ll 1 .
$$

Neglecting again $\omega^{2} \ll k^{2} c^{2}$ and taking the first order in the asymptotic expansion of $Z(f \ll 1) \simeq{ }_{l} \pi^{1 / 2} \exp \left(-f^{2}\right) \simeq{ }_{l} \pi^{1 / 2}$, we find that dispersion relation (4) admits unstable wave solutions with frequency

$$
\omega_{r}=\frac{A}{A+1} \Omega,
$$

which is finite and depends on the magnetic field, and the growth rate 


$$
\omega_{i}=\frac{1}{\pi^{1 / 2}} \frac{k v_{\mathrm{th}, \|}}{A+1}\left[A-\frac{k^{2} c^{2}}{\omega_{p}^{2}}\right],
$$

which has the same form with that of the Weibel instability ${ }^{17}$ in the absence of the stationary magnetic field (and the same limit of small arguments of plasma dispersion function). With no temperature anisotropy, $A=0$, the instability vanishes and Eq. (19) shall define the damping rate, $\omega_{i}<0$. The instability arises for small wave numbers less than a cutoff value

$$
k \leqslant k_{M}=\left(\omega_{p} / c\right) A^{1 / 2},
$$

because the thermal spread effects stabilize first the short wavelength modes. ${ }^{25}$ In this limit, the growth rate is not affected by the presence of the stationary magnetic field, and reaches at the saturation the maximum

$$
\omega_{i}^{\max }=\frac{2}{\pi^{1 / 2} 3^{3 / 2}} \frac{A^{3 / 2}}{A+1} \frac{v_{\mathrm{th}, \|}}{c} \omega_{p}
$$

for

$$
k^{\max }=\sqrt{A / 3}\left(\omega_{p} / c\right) .
$$

The wave frequency in Eq. (18) has no wave-number dispersion, and it has been derived in Ref. 12 as the threshold frequency for the stability of the resonant electron cyclotron waves. Furthermore, Eqs. (18) and (19), as well as the cutoff wave number (20) are derived using the first order approximation of the plasma dispersion function. If we insert Eq. (18) in Eq. (4) and set the imaginary frequency to zero, we find the exact cutoff wavenumber from Eq. (1).

In the limit imposed by the inequality (17), the temperature anisotropy cannot be large. For a small anisotropy, $A$ $\ll 1$, the frequency given by Eq. (18) is in the standard range of the whistler modes, $\omega_{r} \simeq A \Omega \ll \Omega$, and condition (17) is satisfied for parallel temperatures sufficiently large, $v_{\text {th, }}$ $\gg \Omega / k$, which agrees very well with $A<1$. Thus, in the next, we assume a plasma with a small temperature anisotropy, $A=0.2$, and make a comparative evaluation of the exact numerical solutions of Eq. (4). For the whistler mode, the frequency and the growth rate are plotted with dashed lines, and the growth rate of Weibel instability is plotted with a dotted line.

First, in Fig. 6, the ambient magnetic field is considered to be weak, $\Omega / \omega_{p e}=0.01$ (overdense plasma). ${ }^{1}$ In this case the frequency obtained is negligibly small $\left(\omega_{r} \rightarrow 0\right)$, the growth rate approaches quite well that of the Weibel instability in an unmagnetized plasma, and we can assert that the instability develops nonresonantly due to the Weibel mechanism.

For a higher magnetic field, $\Omega / \omega_{p e}=0.1$, the whistler unstable mode becomes resonant with the electrons, and this is confirmed in Fig. 7. In this limit of a small temperature anisotropy, the resonant character of the electromagnetic instability is dominant, provided that the growth rate in Eq. (10) becomes zero in the absence of a longitudinal thermal velocity spread.

We should observe that the frequency of the resonant instability becomes comparable or even larger than the growth rate. This is an empirical criterion we have introduced above in order to discern between the resonant instability that usually has a large frequency, $\omega_{r}>\omega_{i}^{\max }$, and the nonresonant instability with a small frequency, $\omega_{r}<\omega_{i}^{\max }$. Following this principle, we compare the wave frequency (18) with the maximum growth rate (21) and find that the nonresonant instabilities of the Weibel-type will only exist for a background magnetic field sufficiently weak, less than a threshold value given by

$$
\Omega \equiv \frac{|e| B_{0}}{m c}<\frac{2 A^{1 / 2}}{\pi^{1 / 2} 3^{3 / 2}} \frac{v_{\mathrm{th}, \|}}{c} \omega_{p} \simeq 0.033 \frac{v_{\mathrm{th}, \|}}{c} \omega_{p} .
$$

The plasma parameters and the magnetic field chosen in Fig. 6 fulfill this criterion. But as the magnetic field increases and does not satisfy this condition (see Fig. 7), the frequency of oscillations becomes comparable or even larger than the growth rate, $\omega_{r} \gtrsim \omega_{i}$, and the whistler instability becomes resonant.

\section{LIMIT CASE: FILAMENTATION INSTABILITY}

Inspecting the last growth rate in Eq. (13), which does not depend on the parallel temperature but only on the higher perpendicular temperature with respect to the background magnetic field, it is natural to relate the case of a large temperature anisotropy $v_{\mathrm{th}, \perp}^{2} \gg v_{\mathrm{th}, \|}^{2}$, to that of a beam-plasma system, or counterstreaming plasmas moving with a relative streaming velocity $v_{0}$ perpendicular to the stationary magnetic field. Such systems are unstable against the excitation of filamentation instability, which is quite similar to the Weibel instability because both are driven by the particle velocity anisotropy. For propagation along the magnetic field $\left(\mathbf{k} \| \mathbf{B}_{0}\right)$, the filamentation instability is purely growing with the growth rate given by the same Eq. (13) where the streaming velocity $v_{0}$ takes the place of $v_{\mathrm{th}, \perp}$,

$$
\omega_{i}=\omega_{p} \frac{\left[2 k^{2} v_{0}^{2}\left(\omega_{p}^{2}+k^{2} c^{2}\right)-\Omega^{2} \omega_{p}^{2}\right]^{1 / 2}}{2\left(k^{2} c^{2}+\omega_{p}^{2}\right)} .
$$

The dispersion equations (10) and (24) describe now the frequency and, respectively, the growth rate of the filamentation instability assuming two cold and symmetric counterstreaming plasmas, which move perpendicular to the background magnetic field with the streaming velocity $v_{0}$. Such a counterstreaming plasma system can be simply modeled by a monochromatic distribution function $F_{0}=\frac{1}{2} \delta\left(v_{\|}\right)\left[\delta\left(v_{\perp}-v_{0}\right)\right.$ $\left.+\delta\left(v_{\perp}+v_{0}\right)\right]$, and therefore, the case presented here is complementary to that investigated recently in Ref. 26 where the counterstreaming plasmas were considered moving along the static magnetic field, $F_{0}=\frac{1}{2} \delta\left(v_{\perp}\right)\left[\delta\left(v_{\|}-v_{0}\right)+\delta\left(v_{\|}+v_{0}\right)\right]$.

The growth rates of filamentation instability given by Eq. (24) are shown in Fig. 8 for different streaming velocities, and two values of the background magnetic field corresponding to $\Omega / \omega_{p}=0.2$ (long dashed lines) and $\Omega / \omega_{p}=2.0$ (short dashed lines). We have to remark on the same effect of the background magnetic field that limits the existence of filamentation instability to wave numbers larger than a minimum cutoff, 


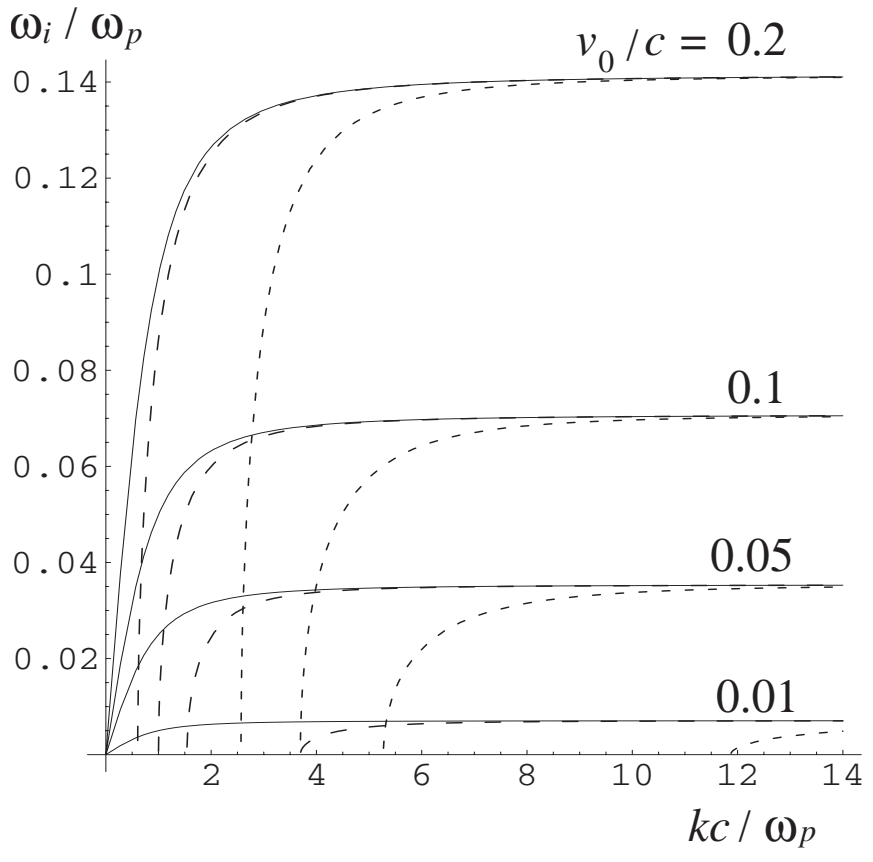

FIG. 8. The growth rates of filamentation instability given by Eq. (24) are shown for two values of the background magnetic field corresponding to $\Omega / \omega_{p}=0.2$ (long dashed lines) and $\Omega / \omega_{p}=2.0$ (short dashed lines). For comparison, with solid lines are shown the growth rates of filamentation instability in an unmagnetized counterstreaming plasma.

$$
k>k_{m}^{f}=\frac{\omega_{p}}{\sqrt{2} c}\left[\left(1+2 \frac{\Omega^{2}}{\omega_{p}^{2}} \frac{c^{2}}{v_{0}^{2}}\right)^{1 / 2}-1\right]^{1 / 2} .
$$

This cutoff wave number vanishes $\left(k_{m}^{f} \rightarrow 0\right)$, in the absence of the background magnetic field (see the solid lines in Fig. 8).

\section{DISCUSSION AND CONCLUSIONS}

We have investigated the unstable modes of the Weibeltype propagating parallel to a stationary magnetic field. The Weibel instability is nonresonantly induced in a plasma with temperature anisotropy due to bunching of the plasma particles by the Lorentz force of the perturbation, with bunches producing a field that enhances the magnetic perturbation. In the presence of a uniform static magnetic field, the free energy stored in the temperature anisotropy $\left(T_{\perp}>T_{\|}\right)$drives the parallel whistler instability, and presently it is mainly believed that the energy is transferred to the whistler mode due to the cyclotron resonance with plasma particles. However, earlier works have proved that the nonresonant mechanism should exist and drive the whistler instability, at least, for anisotropic distribution functions with no parallel thermal velocity spread.

Here we have considered a magnetized plasma with an anisotropic distribution function of the bi-Maxwellian-type. The dispersion relation has been derived in terms of the plasma dispersion function, and in the limits of very large or very small arguments of plasma dispersion function, where we expected to identify the nonresonant whistler instabilities of the Weibel-type. The existence of a large temperature anisotropy in a magnetized plasma is not sufficient for the non- resonant mechanism to be viable, and therefore, in Eq. (2) we have introduced a new criterion for the existence of Weibel instability, which requires for a large perpendicular beta, $\beta_{\perp}>1$. The exact numerical evaluation of the unstable modes has proved that they approach the Weibel-type mode very well when this condition is satisfied. But this criterion has a physical meaning only for a large temperature anisotropy. For small anisotropies we have identified the nonresonant instabilities as having the frequency less or much less than the growth rate, $\omega_{r}<\omega_{i}$. This condition is also satisfied by the nonresonant instabilities driven by a large anisotropy.

In the presence of a stationary magnetic field, the instability is limited not only to a maximum cutoff wave number which depends on the temperature anisotropy, but also by a minimum wave number which depends on the strength of $\mathbf{B}_{0}$. Measuring these cutoff wave numbers allows us to determine the temperature anisotropy and the background magnetic field. Furthermore, the growth rate is clearly reduced by the presence of the ambient magnetic field, and it can be suppressed completely when the stationary magnetic field is sufficiently strong. The critical value, $B_{0, c}$, is given by the equality of the two cutoff wave numbers, $k_{M}=k_{m}$, and from Eqs. (11) and (20) we find

$$
B_{0, c}=\sqrt{8 \pi n m} v_{\mathrm{th}, \|} A \sqrt{A+1},
$$

which, for large temperature anisotropies, becomes

$$
B_{0, c} \simeq \sqrt{8 \pi n m} v_{\mathrm{th}, \perp} A \simeq \sqrt{8 \pi n m} \frac{v_{\mathrm{th}, \perp}^{3}}{v_{\mathrm{th}, \|}^{2}} .
$$

Because the case of a very large velocity anisotropy of plasma particles can be simply correlated to the plasmas flows along the higher temperature direction, we have extended the investigation to the limit of two counterstreaming plasmas. Such a plasma system is unstable against the excitation of the filamentation (Weibel-type) instability, and we have shown that the growth rate of filamentation instability is also diminished by the presence of an ambient magnetic field as was predicted in numerical experiments. ${ }^{21}$ The existence of the filamentation instability is also limited to wave numbers larger than a minimum cutoff, which depends on the strength of the stationary magnetic field.

Finally, we have to remember that this analysis is limited to a linear regime (small amplitudes) of the parallel instabilities of the whistler type. In this case the nonresonant mechanism is strongly competed by the resonant plasma particles, and the small magnetic perturbations required for developing the Weibel instability will be efficient only for an ambient magnetic field sufficiently weak as conditioned by Eq. (2) or Eq. (23). In the second part of this work we shall consider magnetized plasma with a temperature anisotropy $T_{\|}<T_{\perp}$, which drives two nonresonant instabilities: the firehose instability excited along the magnetic filed, and the Weibel instability which propagates in this case perpendicular to the magnetic field. Since it is expected to be much faster than the firehose instability, the Weibel instability would be the primary mechanism of relaxation of the temperature anisotropy. 


\section{ACKNOWLEDGMENTS}

The authors are grateful to the anonymous referee for his insightful comments and the constructive suggestions.

The authors acknowledge financial support from the Research Foundation Flanders-FWO Belgium, the Deutsche Forschungsgemeinschaft through the Sonderforschungsbereich 591, and FOR 1024 through Grant No. Schl 201/21-1. These results were obtained in the framework of Project Nos. GOA/2009/009 (K. U. Leuven), G.0304.07 (FWOVlaanderen), and C 90205 (ESA Prodex 9). Financial support by the European Commission through the SOLAIRE Network (MTRN-CT-2006-035484) is gratefully acknowledged. The numerical results were obtained on the HPC cluster VIC of the K. U. Leuven.

${ }^{1}$ S. P. Gary, Theory of Space Plasma Microinstabilities (Cambridge University Press, Cambridge, 1993).

${ }^{2}$ R. N. Sudan, Phys. Fluids 6, 57 (1963).

${ }^{3}$ K. F. Lee, J. Plasma Phys. 6, 449 (1971).

${ }^{4}$ R. N. Sudan, Phys. Fluids 8, 153 (1965).

${ }^{5}$ N. T. Gladd, Phys. Fluids 26, 974 (1983).

${ }^{6}$ T.-Y. B. Yang, Y. Gallant, J. Arons, and A. B. Langdon, Phys. Fluids B 5, 3369 (1993).
${ }^{7}$ J. Zhao, J.-I. Sakai, and K.-I. Nishikawa, Sol. Phys. 168, 345 (1996).

${ }^{8}$ S. P. Gary and I. V. Cairns, J. Geophys. Res. 104, 19835, DOI: 10.1029/ 1999JA900296 (1999).

${ }^{9}$ S. P. Gary and H. Karimabadi, J. Geophys. Res. 111, A11224, DOI: 10.1029/2006JA011764 (2006).

${ }^{10}$ C. F. Kennel and H. E. Petschek, J. Geophys. Res. 71, 1 (1966).

${ }^{11}$ R. W. Landau and S. Cuperman, J. Plasma Phys. 9, 143 (1973).

${ }^{12}$ J. E. Scharer and A. W. Trivelpiece, Phys. Fluids 10, 591 (1967).

${ }^{13}$ S. P. Gary and C. D. Madland, J. Geophys. Res. 90, 7607, DOI: 10.1029/ JA090iA08p07607 (1985).

${ }^{14}$ S. Hamasaki and N. A. Krall, Phys. Fluids 16, 145 (1973).

${ }^{15}$ S. L. Ossakow, I. Haber, and E. Ott, Phys. Fluids 15, 1538 (1972).

${ }^{16}$ S. Cuperman and Y. Salu, Plasma Phys. 15, 107 (1973).

${ }^{17}$ E. S. Weibel, Phys. Rev. Lett. 2, 83 (1959).

${ }^{18}$ P. H. Yoon and R. C. Davidson, Phys. Rev. A 35, 2718 (1987).

${ }^{19}$ See Section 5.2 in D. B. Melrose, Instabilities in Space and Laboratory Plasma (Cambridge University Press, Cambridge, 1986).

${ }^{20}$ M. Medvedev and A. Loeb, Astrophys. J. 526, 697 (1999).

${ }^{21}$ C. B. Hededal and K.-I. Nishikawa, Astrophys. J. Lett. 623, L89 (2005).

${ }^{22}$ B. D. Fried and S. D. Conte, The Plasma Dispersion Function (Academic, New York, 1961).

${ }^{23}$ R. C. Davidson, D. A. Hammer, I. Haber, and C. E. Wagner, Phys. Fluids 15, 317 (1972).

${ }^{24}$ M. Lazar and R. Schlickeiser, Can. J. Phys. 81, 1377 (2003).

${ }^{25}$ F. Califano, F. Pegoraro, S. V. Bulanov, and A. Mangeney, Phys. Rev. E 57, 7048 (1998).

${ }^{26}$ A. Stockem, I. Lerche, and R. Schlickeiser, Astrophys. J. 651, 584 (2006). 